\title{
A Resource Optimization Framework for Improving Railway-Highway Grade Crossing Safety in Canada
}

\author{
Lalita Thakali \\ Postdoctoral Fellow \\ Department of Civil \& Environmental Engineering \\ University of Waterloo \\ Waterloo, ON, Canada N2L 3G1 \\ Email: 1thakali@uwaterloo.ca
}

\section{Liping Fu (Corresponding author)}

Professor

Department of Civil \& Environmental Engineering

University of Waterloo

Waterloo, ON, Canada N2L 3G1

Intelligent Transportation Systems Research Centre

Wuhan University of Technology

Wuhan, Hubei, China 430000

Tel: 519-888-4567 x 33984

Email: 1fu@uwaterloo.ca

Shahram Heydari

Lecturer

Department of Civil, Maritime and Environmental Engineering

University of Southampton, Southampton, UK

s.heydari@soton.ac.uk 


\section{ABSTRACT}

2 This paper presents a new approach to address the problem of allocating federal resources and 3 identifying upgrading projects for improving the safety of at-grade crossings in Canada. The proposed 4 approach is unique in two key aspects. First, a risk-based network screening process is adopted to 5 identify the priority sites for providing a justifiable basis for distributing the total budget at a regional 6 level as well as narrowing the search space in the subject optimization step. Secondly, a mathematical 7 programming approach is applied to formalize the resource allocation process with explicit 8 consideration of the expected benefits - risk reduction and the costs of implementing the projects. This 9 approach is expected to improve the process of identifying the optimal set of upgrading projects within 10 each region, thus maximizing the return of investment. A full-scale case study from the Canadian 11 crossing network is conducted to demonstrate the application of the proposed approach.

12 Keywords: Grade crossing safety, crash frequency, crash severity, crash cost, resource allocation, 13 resource optimization 


\section{INTRODUCTION}

The Canadian Grade Crossing Improvement Program (GCIP) is a funding program established by Transport Canada about ten years ago to improve safety at high-risk road-railway grade crossings. The GCIP provides contributions of up to $50 \%$ of the eligible costs of crossing upgrades completed by railways and road authorities, such as adding warning signs and signals as well as roadway improvements at crossings (Transport Canada 2015). The average annual funding provided under this program was approximately 11.7 million Canadian dollars from 2009 to 2014. Apart from the GCIP funding, Transport Canada also has other complementary funding programs such as the Grade Crossing Closure Program (GCCP) and Asia-Pacific Gateway and Corridor Initiative (APGCI) for funding grade crossing closure and grade separation projects, respectively. Recently, Transport Canada has announced the Rail Safety Improvement Program (RSIP) to replace the GCIP with a similar funding mechanism but with larger goal of improving overall rail safety and reducing injuries and fatalities related to rail transportation in Canada.

Currently, Transport Canada adopts a bottom-up approach to allocate the federal GCIP budget where railways and road authorities first submit their applications for individual upgrading projects that are deemed necessary. Then eligible projects are often identified based on their characteristics and some selection criteria. Often some applicants (concerned authorities) are not aware of the available funding or have difficulty to identify the crossing sites for upgrading (Transport Canada 2015). Therefore, Transport Canada's regional inspectors are encouraged to help the local authorities in identifying and discussing potential projects to seek funding from the federal government. One of the weaknesses of this current practice is that the process of identifying the potential upgrades is usually based on a relatively limited cost-benefit analysis. As a result, there is no guarantee that the federal budget is utilized to an optimum level.

One of the approaches to dealing with this resource (budget) allocation challenge could be targeting the highest-risk locations obtained through the standard network screening process. The network screening process provides a systematic method to identify a list of locations with high collision risk by considering risk factors (e.g., site characteristics, control type, site specific collision history, exposure level and so on) associated with each crossing. While this process can help determine the candidate crossings for safety improvement, it does not address the question on which treatments should be applied for achieving the maximum cost benefit. The fact that there could be multiple alternative countermeasures for improving safety of a given crossing, the cost and safety benefit associated with each option may differ accordingly. For example, a passive crossing could be upgraded to FLB (a crossing with flashing lights and bells) or FLBG (a crossing with flashing lights, bells and gates) with the FLB control device costs relatively less than the FLBG. However, the former also is expected to have a lower effectiveness in reducing collision risk than the former. Therefore, one needs to assess both the safety effect and the costs while selecting a countermeasure. At an individual project level, a simple cost-benefit analysis could be helpful to select the appropriate treatment option (Volmer et al. 2006; Cooper et al. 2007; Rezvani et al. 2015; Fu et al. 2016). However, when it comes to allocating resources by a federal government at its regional network level, a holistic approach considering the overall benefits is required. To this end, the resource allocation problem arises.

There are two main challenges in dealing with resource allocation problem for a country like Canada with a federal government structure. First, it is important to ensure that the funding from the federal government is allocated fairly across its various regions, i.e., provinces and territories. If only the top high-risk crossings from the standard network screening process, which only considers crash frequency, are considered, some regions may not receive any funds due to relatively low exposure or risk overall. For a discussion in this regard, see Heydari et al. (2018). Second, once there is a certain amount of 
resource to be allocated to a region, there is a need for a systematic process to identify a list of potential upgrades. The purpose of this study is to develop a comprehensive approach for allocating resources between and within regions. An optimization approach is introduced to identify upgrades with the objective of achieving the maximum safety benefit under a given budget constraint.

\section{LITERATURE REVIEW}

The resource allocation problem arising in grade crossing safety improvement programs involves selecting the candidate sites and the safety improvement project or countermeasure for each site across a railway grade crossing network. At the simplest level, this problem can be looked site-by-site where alternative countermeasures for upgrading individual sites are identified on the basis of some specific measures of effectiveness, such as benefit cost ratio and net present value. For example, Volmer et al. (2006) applied benefit-cost ratio for project selection to improve the safety of grade crossings in Iowa. Similarly, Rezvani et al. (2015) employed a benefit-cost approach to determine if a proposed treatment option is justifiable for the safety improvement of a given grade crossing considering all public grade crossings across North Carolina. A similar approach was considered by Cooper et al. (2007) for safety improvement of grade crossings in California. Fu et al. (2016) in their recent project submitted to Transport Canada applied benefit-cost ratio and net present value for selecting appropriate countermeasures. The motive behind all these studies is to identify the best safety treatment options for a given set of crossings such that the funding (resource) could be utilized effectively.

Similar concept is also used by the US Department of Transportation (DOT) in its resource allocation procedure for highway-rail grade crossing improvements, which was developed to assist its states and railroad agencies in determining the effective allocation of federal funds for crossing traffic control improvements (Farr 1987). It makes use of two analytical tools, namely, accident prediction model and resource allocation model. All crossings with either passive or flashing light warning devices are considered as the candidates for upgrading and collision risk associated with these crossings are determined by accident prediction model. For illustration purpose, three improvement options - passive to flashing lights, passive to gates and flashing lights to gates, have been considered in the DOT's guideline. Each of these options has its own cost and safety effectiveness which are used to calculate the safety benefit of each treatment at each site. Then, benefit-cost ratio is computed for each option and site; and consequently, the treatment option with the highest benefit-cost ratio is considered. Once this is repeated for all the sites under investigation, they are ranked based on the benefit-cost ratio. The sites are considered for improvement from the ranked list until the budget reaches to its limit. It is noted that this ranking-based approach is a heuristic approach, and therefore, the solution achieved may not necessarily be optimum. It is also noted that the US DOT's resource allocation procedure does not dictate the final decision for crossing improvements, rather it recommends for further diagnostic process to aid in making a final decision.

Alternatively, the resource allocation problem can be addressed by formulating an optimization model aiming at maximizing expected total safety benefits from improving a given set of grade crossing sites. Unlike the previously mentioned approach, this method provides a network-based approach where all crossings in a given network are considered collectively rather than individually. The scheme of network-wide resource allocation optimization is quite commonly proposed in various transportation engineering fields such as pavement engineering (e.g., Hardwood et al. 2003; Shohet and Perelstein 2004), and road safety studies (e.g., Kar and Datta 2004; Banihashemi 2007; Mishra and Khasnabis 2012). However, this scheme is rarely applied for allocating resources for improving safety at grade crossings with the exception of Konur et al. (2012). We demonstrated the use of an optimization model for allocating the resource for improving grade crossing safety in a case study from Tennessee. In their study, the collision risk associated with each crossing site was estimated using US DOT's accident 
prediction models. The study compared results from the US DOT's resource allocation approach and the proposed optimization model and concluded that the latter provided better performance in terms of collision risk at the crossing network level. Note that Konur et al.'s study on applying an optimization approach to resource allocation represents a regional level problem. For a federally funded program such as GCIP, it is a critical requirement to consider both between and within region levels in making resource allocation decisions.

\section{3. METHODOLOGY}

8 Figure 1 shows our proposed resource optimization framework for allocating grade crossing safety improvement funds at a regional level and selecting specific crossings within each region with their proposed treatment options. The framework integrates a risk-based approach with two crash risk models; collision frequency and severity models. A Negative Binomial (NB) collision frequency model has been developed for each crossing type (i.e., crossing with a standard reflectorized crossing sign (hereafter SRCS), crossing with SRCS and stop sign (hereafter SRCS+STOP), FLB and FLBG). The NB model provides long-term estimates of collision frequencies which are then adjusted by applying the Empirical Bayes (EB) approach (Hauer 1997), one of the most widely used approaches by transportation professionals in safety analysis. A multinomial logit model has been calibrated for the severity model to estimate the probability of a given type of collision severity (i.e., fatal, serious injury, minor injury and property damage). As illustrated in Figure 1, our resource allocation framework has three main components: 1) identify priority sites across the given grade crossing network, 2) allocate resource (budget) at regional level, and 3) allocate resource at region's network level. Outputs from each component become input to the latter. The following provides a brief description on each component.

\subsection{Identification of Priority Sites}

In this component, a list of priority sites that represent the potential candidates for safety improvement are identified. For this, we follow the standard procedure of network screening, which is commonly used in the transportation safety management process. This component serves two purposes. First, the total available funds can be distributed across the regions/provinces based on the number of priority sites identified in each region. Second, by considering only the priority crossings identified, we can narrow our search in selecting upgrades within each region, which will significantly reduce computation time in the optimization process (the third component of the framework). The following steps are proposed for identifying the priority sites:

a. Estimate collision frequency: We first estimate the expected collision frequency associated with each site. For this, we employ an EB approach. The fundamental idea behind the EB method is to obtain an estimate of collision frequency at a given site by combining observed collisions at that site and predicted collision frequency from a cross-sectional model that is used to reflect risk due to the site-specific conditions. These two safety measures are combined by using a weight parameter which depends on the reliability of the cross-sectional model. The cross-sectional model used in this study is the NB model and is calibrated using a sample dataset that consists of collision counts as a dependent variable and site conditions, such as traffic volumes, geometric and operational characteristics as predicting variables.

The expected collision frequency from the EB method at a given site $\mathrm{i}$, denoted as $\mathrm{Ni}$, is given as follows:

$N_{i}=w_{i} \mu_{i}+\left(1-w_{i}\right) y_{i}$ 
where,

- $w_{i}$ is a weight given by $\frac{1}{1+\alpha \mu_{i}}$

- $y_{i}$ is observed collision frequency at site $\mathrm{i}$;

- $\mu_{i}$ is predicted collision frequency at site $\mathrm{i}$ and is obtained from a NB model;

- $\alpha$ is dispersion parameter of the NB model, which is obtained as part of the process of calibrating the model.

The time period for the observed and predicted collision frequencies has to be same. Note that if the unit of $\mathrm{N}_{\mathrm{i}}$, is other than the collision frequency per year, it will be normalized accordingly.

b. Estimate collision severity: When a collision happens, it could result into various severity types depending on their level of consequences. For example, it could be a fatal collision, serious injury, minor injury or property damage only. We estimate the probability associated with each of these severity types employing a multinomial logit model as given below:

$$
P_{i j}=\frac{e^{Z_{i j}}}{\sum_{j=1}^{4} e^{Z_{i j}}}
$$

where,

- $\quad \mathrm{i}$ is an index representing a given site $\mathrm{i}$;

- $\quad \mathrm{j}$ is an index representing a collision severity type with $\mathrm{j}=\mathrm{FI}$, SI, MI, and NI for fatal injury, serious injury, minor injury, and no injury, respectively;

- $\quad P_{i j}$ is an estimate of probability of severity type $\mathrm{j}$ for site $\mathrm{i}$ and;

- $Z_{i j}$ is a measure representing the propensity for a collision at crossing $\mathrm{i}$ to experience severity type $\mathrm{j}$.

A multinomial logit model is calibrated using a sample dataset that consists of collision severity type as a dependent variable and site conditions, such as traffic volumes, physical characteristics, operational characteristics as predicting variables.

c. Estimate total risk: Total risk is calculated by multiplying the expected number of collisions obtained from Step 1 by the corresponding probability of each consequence type obtained from Step 2. Note that each severity type is converted to a dollar value by assuming a cost associated with it. Mathematically, total risk for a given site i is given as follows:

$$
R_{i}=N_{i} \sum_{j=1}^{4} P_{i j} C S_{j}
$$

where,

- $\quad \mathrm{i}$ an index representing a given site $\mathrm{i}$;

- $\mathrm{j}$ is an index representing a collision severity type, where the subscripts $1,2,3$ and 4 denotes fatal injury, serious injury, minor injury and no injury, respectively;

- $N_{i}$ is expected annual collision frequency from Step 1

- $P_{i j}$ is an estimate of probability of severity type $\mathrm{j}$ for a given site $\mathrm{i}$ from Step 2, and

- $C S_{i j}$ is cost associated with the severity type $\mathrm{j}$. 
d. Select priority sites: After estimating total risk associated with each site using Eq 3, the sites are ranked based on the magnitude of their total risk. Finally, the top M riskiest sites (or represented in certain percentage) is selected as the priority sites.

\subsection{Allocation of Resources at a Regional Level}

To allocate federal budget at the regional level, we follow a risk-based approach. We first look at the distribution of the number of priority sites falling under each region. From the previous discussion, we know that the priority sites are generated through the risk assessment. The percentage of budget to be allocated to a given region is then set to be the same as the proportion of the priority sites falling within that region. Mathematically, the federal budget to be allocated to a region can be calculated as follows.

$$
\mathrm{B}_{r}=B^{T} \frac{m_{r}}{M}
$$

where,

- $\mathrm{M}$ is the total number of sites in the priority list;

- $\mathrm{m}_{\mathrm{r}}$ is the number of sites included in the priority list of the region $\mathrm{r}$;

- $\mathrm{B}^{\mathrm{T}}$ is the total federal budget available for the analysis year $\mathrm{T}$; and

- $\mathrm{B}_{\mathrm{r}}$ is the total calculated budget for the region $\mathrm{r}$.

\subsection{Identification of Upgrades within a Region}

After determining the amount of federal budget to be allocated to each region, the next step is to select specific safety improvement projects within each region. For this task, it is necessary to first identify the potential or applicable countermeasures for each candidate crossing, and then find the list of upgrades that would maximize the total safety benefit while satisfying the budget constraint. This could be formulated as an optimization problem for which the improvement sites and their upgrading options are identified such that optimum benefit is achieved within each region. A mathematical model is formulated where the key parameters are budget limitation and countermeasure options (FLB, FLBG with gates, etc.) with their costs and safety effectiveness values. In this research, the benefit refers to the annual reduction in collision costs. It should be noted that other benefits such as delay reduction and environmental benefits could be easily incorporated into our optimization framework when the required data and models become available.

\subsection{Model Formulation}

Let I' $=\{1 \ldots \mathrm{I}\}$ be the set of crossing priority sites for a given region and the sites be indexed by $i$. Let $\mathrm{K}^{\prime}=\{1 \ldots \mathrm{K}\}$ be the potential set of countermeasures that could be used to treat the sites and the countermeasure be indexed by $\mathrm{k}$. Each countermeasure $\mathrm{k}$ has a specific implementation cost denoted by $C C_{k}$ and safety effectiveness value denoted by $S E_{k}$. Safety effectiveness is often referred to as collision modification factor in road safety literature. It is a relative term that compares risk after and before the implementation of a countermeasure. In other words, this measure quantifies by how much risk is expected to decrease when a selected countermeasure is applied. These values are obtained from a separate study.

Let $S B_{i k}$ be the annual safety benefit expected by implementing countermeasure $\mathrm{k}$ at a given site $\mathrm{i}$. It is measured in terms of reduction in the expected total risk after the upgrade, which is determined using total risk $\left(R_{i}\right)$ and safety effectiveness $\left(S E_{k}\right)$ as follows: 
$1 \quad S B_{i k}=R_{i} *\left(1-S E_{k}\right)$

2 where,

$8 z_{i k}=\left\{\begin{array}{l}1, \text { if countermeasure } k \text { can be implemented at site } i \\ 0, \text { Otherwise }\end{array}\right.$

- $S E_{k}$ is safety effectiveness of countermeasure $\mathrm{k}$. $z_{i k}$ as:

- $\quad R_{i}$ is expected annual total risk before the implementation of countermeasure k;

It is obvious that not all countermeasures can be applied to every crossing site for some technical constraints may exist, for example, due to specific site characteristics. Thus, we define a binary variable

$z_{i k}$ values are pre-determined by looking at the characteristics of a given crossing site. For example, let 10 a crossing has a passive control with signs only. The potential countermeasures be converting passive 11 to FLB crossing, converting passive to FLBG crossing and adding gates to FLB crossings. Then for this 12 passive crossing, the last countermeasure is not applicable and thus its $z_{i k}$ value becomes zero.

13 Similarly, not all potential countermeasures represented in the list $K$ can be implemented due to the budget constraint. Through the optimization, we determine which countermeasure to implement to which crossing site. To represent this decision, we use a binary variable $x_{i k}$ which is defined as follows:

$x_{i k}=\left\{\begin{array}{l}1, \text { if countermeasure } k \text { is implemented at site } i \\ 0, \text { Otherwise }\end{array}\right.$

From the above description, it follows that $x_{i k} \leq z_{i k}$.

19 The total budget needed for implementing the selected countermeasures becomes

$20 \quad \sum_{i=1}^{I} \sum_{k=1}^{K} C C_{k} \times x_{i k}$

Let $B_{r}$ be the available budget for improving the crossings in a given region for the given year. Then, there exist a constraint such that $\sum_{i=1}^{I} \sum_{k=1}^{K} C C_{k} \times x_{i k} \leq B_{r}$.

23 The total safety benefit is $\sum_{i=1}^{I} \sum_{k=1}^{K} S B_{i k} \times x_{i k}$

The mathematical model for the resource allocation can be summarized as follows:

$$
\text { maximize } \mathrm{SB}=\sum_{i=1}^{I} \sum_{k=1}^{K} S B_{i k} \times x_{i k}
$$

Subject to:

$$
\begin{aligned}
& \sum_{i=1}^{I} \sum_{k=1}^{K} C C_{k} \times x_{i k} \leq B_{r} \\
& \sum_{k=1}^{K} x_{i k} \leq 1, \quad i=1, \ldots, I
\end{aligned}
$$

where,

$$
\begin{aligned}
& x_{i k} \leq z_{i k}, \quad i=1, \ldots, I ; \quad k=1, \ldots, K \\
& x_{i k} \in\{0,1\}, \quad i=1, \ldots, I ; \quad k=1, \ldots, K
\end{aligned}
$$


1 In the formulation (Eq. 8-12), the objective is to maximize the total expected safety benefit (SB), as given by Eq. 8. Eq. 9 is the budget constraint while Eq. 10 is a policy constraint which we assume here that at most one countermeasure can be implemented at each site. This assumption can be relaxed when it is necessary. Eq. 11 controls the list of countermeasures being considered for implementation at specific crossings. Eq. 12 is a constraint that allows the binary variable $x_{i k}$, an indicator for potential countermeasure $\mathrm{k}$ at site $\mathrm{i}$, to take value 1 or 0 where 1 means it is selected for implementation and 0 otherwise.

\section{CASE STUDY: APPLICATION TO CANADIAN GRADE CROSSING NETWORK}

\subsection{Network Description}

This section discusses a practical application of the proposed methodology - allocation of safety improvement budget across Canadian provinces and territories and their corresponding crossing network. Canada currently has a total of 26,640 federal regulated railway-highway crossings, of which $90 \%$ are grade crossings that are controlled by passive and active warning devices. While all crossings must be guided with a minimum level of warning using passive warning devices such as crossbucks and stop signs, some crossings also warrant for active devices such as FLB and FLBG or even grade separation when the exposures (trains, vehicles and pedestrians) are relatively high and safety is a concern. Of the 26,640 total crossings, 16,650 crossings (60.4\% passive and $39.6 \%$ active) have complete information on some important crossing attributes (those that are needed for risk estimation) and are thus considered in this case study (Table 1). These crossings are located in nine provinces (Saskatchewan, Ontario, Alberta, Manitoba, Quebec, British Columbia, New Brunswick, Nova Scotia and Newfoundland \& Labrador) and two territories (Yukon and North West), as shown in Figure 2. Our objective is to distribute a typical annual federal safety budget across these regions, and for each region, identify the most hazardous crossings and the respective upgrades. The total budget is assumed to be 13 million Canadian dollars.

\subsection{Selected Countermeasures}

Four types of major countermeasures are considered in our case study, as listed in Table 2. Note that the option of grade-separation was not included in this analysis but can be readily considered in our proposed framework. The type of countermeasures eligible for a given crossing depends on the existing conditions of the crossing. For example, conversion of passive to FLB crossing only makes sense for a crossing with passive control type (e.g., SRCS or SRCS with STOP signs). Similarly, adding gates to FLB crossing is applicable only for FLB crossings. Therefore, one must first gain a full knowledge about the existing conditions of the crossings and carefully examine the validity of each countermeasure before considering them as a potential treatment option. For the countermeasures selected in this study, the possible treatment options for each crossing follows the following logic:

As mentioned in section 3, for our case study, only a single treatment option is allowed for each site. However, the optimization program can easily be modified to accommodate multiple options. Table 2 shows safety effectiveness and cost associated with the corresponding countermeasures. The safety effectiveness of the abovementioned interventions was obtained from a previous study conducted by

1. If a crossing is passive, treatment options are FLB and FLBG

2. If a crossing is FLB, treatment option is FLBG

3. If a crossing is FLBG, treatment option is adding constant warning device (CWD) Fu et al. (2016). The costs were supplied by Transport Canada. 


\subsection{Safety Models}

As discussed previously, safety models - collision frequency and collision severity models - are required for the resource allocation framework. These models were recently updated using the historical data from 2011 to 2016. Eq. 13 to Eq. 16 present models for four different crossing types - SRCS, SRCS with STOP sign, FLB and FLBG, respectively. The first two belongs to passive crossing category and the latter two to active crossing category. Similarly, Eq. 17 presents the collision severity model.

$$
\begin{aligned}
& \mu^{S R C S}=e^{-7.866+0.517 * \ln (T E)+0.012 * T S+0.011 * R S+0.591 * U} ; \alpha=2.262 \\
& \mu^{S R C S+S T O P}=e^{-5.810+0.471 * \ln (T E)+0.083 * \mathrm{RG}} ; \alpha=2.342
\end{aligned}
$$$$
\mu^{F L B}=e^{-8.158+0.276 * \ln (T E)+0.013 * T S+0.014 * \mathrm{RS}+0.585 * U+0.808 * W C+0.313 * N L+0.274 * N T} ; \quad \alpha=1.131
$$

$$
\mu^{F L B G}=e^{-5.647+0.325 * \ln (T E)+0.278 * \mathrm{WC}+0.057 * \mathrm{RG}} ; \quad \alpha=1.880
$$

where,

- $\mu^{S R C S}, \mu^{S R C S+S T O P}, \mu^{F L B}$ and $\mu^{F L B G}$ are predicted collision frequencies over a six-year time period for SRCS, SRCS with STOP, FLB and FLBG crossings, respectively;

- TE is total traffic exposure defined as the product of the Average Annual Daily Traffic (AADT) and the number of trains traversing the crossing per day;

- TS is weighted maximum train speed $(\mathrm{km} / \mathrm{hr})$, which is the average of the maximum speeds of freight trains, passenger trains and switch trains weighted by their daily traffic;

- $\quad$ RS is road speed limit $(\mathrm{km} / \mathrm{hr})$;

- $\mathrm{U}$ is location indicator, $\mathrm{U}=1$ if the crossing is located in an urban area otherwise 0 ;

- NT is number of tracks at the crossing;

- NL is number of lanes at the crossing; and

- $\mathrm{RG}$ is average road gradient (\%); and

- WC is whistle cession; and

- $\alpha$ is dispersion parameter of the NB model,

$$
P_{i j}=\frac{e^{Z_{i j}}}{\sum_{j=1}^{4} e^{Z_{i j}}}
$$

$$
\text { where, }
$$

- $\mathrm{Z}_{i j}= \begin{cases}-2.935+0.026 * T S-0.012 * R S+0.092 * R G, & j=F I \\ -2.412+0.012 * T S-0.003 * R S+0.050 * R G, & j=S I \\ -2.171+0.004 * T S+0.006 * R S+0.074 * R G, & j=M I \\ 0 & j=N I\end{cases}$

- $\mathrm{TS}=$ Maximum train speed $(\mathrm{km} / \mathrm{hr}) ;$ and

- $\quad \mathrm{RS}=$ Road speed limit $(\mathrm{km} / \mathrm{hr})$

- $\mathrm{RG}=$ Average road gradient $(\%)$

Note that the expected collision frequency obtained from collision models presented here from Eq. 13 to Eq. 16 represent risk over the six-year period. It was, therefore, converted to their annual measures by dividing these estimates by six.

Table 3 presents the equivalent monetary collision costs of individual severity types. These values were suggested by Transport Canada to convert collisions to their monetary costs. 


\subsection{Discussion of Results}

The optimization problem described in section Model Formulation is coded in R platform ( $\mathrm{R}$ Core Team 2017) and solved using GLPK solver available in the R package. Since this is an integer programming problem, it is solved using branch and bound algorithm.

\subsubsection{Resource Allocation at a Regional Level}

As described in Methodology, the first task in the resource allocation process is to identify the priority sites within the selected network. For this case study, we considered the top 10\% high risk crossings (i.e., 1665 sites) across Canada as the priority sites. The location of these sites is shown in Figure 3. To estimate the risk of each site, the inventory database that records site-specific physical and operation characteristics (e.g., number of tracks, number of lanes, traffic volumes, train speed limit, road speed limit, and others) of passive and active crossing are utilized. Then, total risk associated with each site is estimated using the collision frequency and severity models we calibrated previously.

The number of priority sites in terms of its percentage across the regions (i.e., nine Canadian provinces and two territories) is calculated and is shown in Figure 4 (a). Among them, Ontario has the highest number of priority sites $(42.22 \%)$ while Yukon and Northwest territories has none. Next, the assumed federal budget of 13 million dollars for the given year is distributed across the regions based on the percentage of priority sites falling within each region. Accordingly, Ontario gets the highest share of the budget as shown in Figure 4 (b).

\subsubsection{Identification of Upgrading Projects within Each Region}

The process of identifying upgrading projects within each region involves two steps. The first step is determining priority sites and budget cap for each region, as explained previously. This is followed by the second step of applying an optimization model to identify the upgrading sites along with the treatment options under the prespecified budget.

The example results of this two-step approach (hereafter referred as RA ${ }^{\text {opt }}$-I) for the two selected provinces (Ontario and Alberta) are summarized in Tables 4 and 5 and Figure 5. Based on this approach, Ontario has a total of 703 grade crossings that were identified as priority sites. Accordingly, the budget available for Ontario is 5.48 million dollars. After optimization, a total of 91 sites were identified as the candidate sites for safety improvement. A summary of selected countermeasures is presented in Table 4. From the analysis, the first-year safety benefit by upgrading 91 sites in Ontario is expected to be 5.74 million dollars. Considering a service life of 20 years. the expected total safety benefit would be 114.74 million dollars. This represents a rough estimate based on the assumption that the safety benefits from upgrading the site remain constant in the future year (assuming no changes in the crossing conditions).

Similarly, for Alberta, there is a total of 235 grade crossings identified as priority sites and the total budget available is 1.83 million dollars. Among them, 30 sites were identified as safety improvement sites and the expected annual and total safety benefits are 1.47 and 29.47 million dollars, respectively (Table 4). Figure 5 (a) and (b) show the location of the selected sites labelled with their treatment option for Ontario and Alberta, respectively.

For the sake of benchmarking, we also solved this resource allocation problem following two other approaches. The first approach considered is the ranking method based on benefit-cost ratio, hereafter referred as RA ${ }^{\text {rank}}-$ II. Similar to the RA ${ }^{\text {opt }}$-I approach, RA $^{\text {rank}}-$ II also includes two steps: a) budget is first allocated at the regional level, which is same as the $\mathrm{RA}^{\mathrm{opt}}-\mathrm{I}$; and b) sites for upgrading are then identified for the given budget limit. Unlike the optimization approach, this ranking method considers the top high-risk crossings site-by-site and determines the best options for upgrading individual sites 
using benefit cost ratio. The sites are selected from the ranking list until the budget is exhausted. From our analysis as shown in Table 5, we found that the ranking approach did not always meet the optimal condition of achieving maximum safety benefit for most of the provinces. For example, Alberta, Quebec, Saskatchewan, Manitoba and British Columbia show lower safety benefit compared to the RA ${ }^{\text {Opt }}$-I approach for the same respective budget limit. Also, the overall total benefit appears to be slightly higher for $\mathrm{RA}^{\mathrm{opt}}$-I (approximately $\$ 4.6$ million). This comparison analysis clearly shows that the optimization approach is better than the traditional ranking method based on benefit-cost ratio.

9 Second, we run the optimization model to allocate the same total budget of 13 million dollars across 10 Canada by considering the entire set of priority crossings (i.e., 1665) together. This one-step approach is referred here as RA ${ }^{\text {opt }}$-III. One of the limitations of this approach, as expected, is that it resulted in a less balanced distribution of funds across the individual provinces, as shown in Table 5. For example, Ontario has more resource allocated with 102 sites selected for upgrading whereas New Brunswick and Nova Scotia has none. Also, this approach required significantly more computational time. In terms of achieving overall total safety benefit, this approach performed slightly better.

\subsubsection{Sensitivity Analysis}

We also conducted a sensitivity analysis to investigate how the total safety benefit over the project service-life period of 20 years vary with the amount of investment made by the federal government. This analysis could be useful for the government to gain a clear picture of relationship between the funding level and the expected total safety benefit and thus make more informed investment decisions. For this analysis, the budget was set to increase from 2 million dollars to 20 million dollars at an increment of 2 million dollars and the resource allocation procedure (RA ${ }^{\text {opt }}$-III) was applied to determine the total safety benefit for the each given budget. As mentioned previously, for a given project life cycle, its annual safety benefit is assumed to remain constant for all the years. From the analysis shown in Figure 6, the total safety benefit is found to increase with the budget level, which is expected. By setting a target level for safety benefit, the federal government can figure out the amount of investment required.

\section{CONCLUSIONS}

This study proposed an optimization framework to allocate a given federal budget across and within different regions. This approach allows us to determine countermeasures specific to crossing sites for upgrading while maximizing the overall safety benefit of a given rail network considering the available budget limit. A case study is used to show the application of the proposed project using the grade crossing network of Canada, demonstrating the promising value of the proposed framework. Note that the resource allocation approach proposed in this study is intended to include only major upgrades; and therefore, the allocation of regular maintenance budget is recommended to be considered separately as currently practised by Transport Canada. Also, it is noted that the case study presented in this study is sensitive to the quality of the input data and parameters, such as crossing attributes and the cost and the safety effectiveness of countermeasure, the accuracy of the safety models and the unit costs of different types of collisions. Further research is needed to examine the sensitivity of the model to these inputs and parameters. Further improvements would be achieved by employing more advanced statistical methods such as those that account for dependencies among differing injury-severity levels (e.g., mixed logit model) and those that accommodate spatial dependencies in the data.

The authors wish to acknowledge Transport Canada for their financial supports and providing the data. 


\section{REFERENCES}

Banihashemi, M., 2007. Optimization of highway safety and operation by using crash prediction models with accident modification factors. Transportation Research Record 2019, Transportation Research Board, Washington.

Cooper, D.L., MacLeod, K.E. and Ragland, D.R., 2007. Rail crossings: a strategy to select countermeasure improvements for rail-highway crossings in California. Tech. Rep. Safe Transportation Research \& Education Center, UC Berkeley. Retrieved from: http://escholarship.org/uc/item/8fp8t8zs.

Farr, E.H., 1987. Rail-Highway Crossing Resource Allocation Procedure - User's Guide, Third. Edition. U.S. Department of Transportation, Federal Railroad Administration, Washington, DC.

Fu, L., Heydari, S. and Thakali, L., 2016. Improvement and Development of Collision Risk and Treatment Effectiveness Models for GradeX. Submitted to Transport Canada.

Fu. L., 2018. GradeX - A Decision Support Tool for Improving Safety at Highway- Railway Grade Crossings in Canada. Submitted to Transport Canada.

Harwood, D., Rabbani, E., and Richard, K., 2003. Systemwide optimization of safety improvements for resurfacing, restoration, or rehabilitation projects. Transportation Research Record, Transportation Research Board, (1840), 148-157.

Hauer, E., 1997. Observational before/after studies in road safety. Estimating the effect of highway and traffic engineering measures on road safety.

Heydari, S., Fu, L., Thakali, L. and Joseph, L., 2018. Benchmarking regions using a heteroskedastic grouped random parameters model with heterogeneity in mean and variance: applications to grade crossing safety analysis. Analytic Methods in Accident Research 19, 33-48.

Kar, K., and Datta, T. K., 2004. Development of a safety resource allocation model in Michigan. Transportation Research Record, Transportation Research Board, No. 1865, Washington, DC, 6471.

Konur, D., Golias, M. M., and Darks, B., 2013. A mathematical modeling approach to resource allocation for railroad-highway crossing safety upgrades. Accident Analysis and Prevention, 51, 192-201.

Mishra, S., and Khasnabis, S., 2012. Optimization Model for Allocating Resources for Highway Safety Improvement at Urban Intersections. Journal of Transportation Engineering, 138(5), 535-547.

R Core Team, 2017. R: A language and environment for statistical computing. R Foundation for

Statistical Computing, Vienna, Austria. URL https://www.R-project.org/.

Rezvani, A. Z., Peach, M., Thomas, A., Cruz, R., and Kemmsies, W., 2015. Benefit-Cost methodology for highway-railway grade crossing safety protocols as applied to transportation infrastructure project prioritization processes. Transportation Research Procedia, 8, 89-102.

Shohet, I. M., and Perelstein, E., 2004. Decision support model for the allocation of resources in rehabilitation projects. Journal of Construction Engineering and Management, 130(2), 249-257. 
Transport Canada, 2015. Evaluation of the Grade Crossing Improvement Program. Final Report, Evaluation and Advisory Services, Transport Canada. https://www.tc.gc.ca/eng/corporateservices/des-reports-1268.html [Access in January 2018].

Volmer, N., Baer, P., Gibson, J., Hey, J., Lutz, W., O’Riley, C. and McCauley, D., 2006. Federal-aid railroad-highway grade crossing program: use of a benefit-cost ratio to prioritize projects for funding. Tech. Rep., Office of Rail Transportation, Modal Division, Iowa Department of Transportation. 
Table 1: Summary of crossing sites

\begin{tabular}{|c|c|c|c|}
\hline \multicolumn{2}{|c|}{ Crossing type } & No of crossings & Crossings in percentage \\
\hline \multirow{2}{*}{ Passive crossing } & SRCS & 7943 & 47.71 \\
\hline & SRCS + STOP & 2115 & 12.70 \\
\hline \multirow{2}{*}{ Active crossing } & FLB & 4104 & 24.65 \\
\hline & FLBG & 2488 & 14.94 \\
\hline To & & 16650 & 100 \\
\hline
\end{tabular}

Table 2: Safety effectiveness and cost for countermeasures

\begin{tabular}{clcc}
\hline S.N. & Countermeasure & $\begin{array}{c}\text { Cost } \\
\text { (in thousand dollars) }\end{array}$ & $\begin{array}{c}\text { Safety Effectiveness } \\
\text { (SE) }\end{array}$ \\
\hline 1 & Passive to FLB & 60 & 0.395 \\
\hline 2 & Passive to FLBG & 80 & 0.297 \\
\hline 3 & Adding gates to FLB & 50 & 0.647 \\
\hline 4 & Adding CWD to FLBG & 60 & 0.571 \\
\hline
\end{tabular}

Table 3: Collision costs based on severity types

\begin{tabular}{cll}
\hline S.N. & Collision severity type & Cost per collision \\
\hline 1 & Fatal collision & 7.04 million dollars \\
\hline 2 & Serious injury collision & 0.405 million dollars \\
\hline 3 & Minor injury collision & 0.205 million dollars \\
\hline 4 & No injury collision & 16,800 dollars \\
\hline
\end{tabular}


Table 4: Summary results of resource allocation for Ontario and Alberta (RA $\left.{ }^{\mathrm{opt}}-\mathrm{I}\right)$

\begin{tabular}{llc}
\hline Crossing type & Countermeasure & No of selected sites \\
Active (FLB) & Province: Ontario & \\
Active (FLBG) & FLB to FLBG & 2 \\
Passive (SRCS or SRCS+STOP) & Add CWD to FLBG & 76 \\
Total sites & Passive to FLB & 11 \\
Budget (million \$) & Passive to FLBG & 2 \\
Annual safety benefit (million \$) & & $\mathbf{9 1}$ \\
Total safety benefit (million \$) & 5.489 & \\
& 5.74 & \\
Active (FLB) & 114.74 & 1 \\
Active (FLBG) & Province: Alberta \\
Passive (SRCS or SRCS+STOP) & FLB to FLBG \\
Total sites & Add CWD to FLBG \\
Budget (million \$) & Passive to FLB & 17 \\
Annual safety benefit (million \$) & Passive to FLBG & 2 \\
Total safety benefit (million \$) & & $\mathbf{3 0}$ \\
\hline
\end{tabular}

Table 5: Comparison of resource allocation approaches

\begin{tabular}{|c|c|c|c|c|c|c|c|c|c|}
\hline \multirow{2}{*}{ Provinces/Territories } & \multicolumn{3}{|c|}{ Number of selected sites } & \multicolumn{3}{|c|}{$\begin{array}{l}\text { Annual safety benefit } \\
\text { (million \$) }\end{array}$} & \multicolumn{3}{|c|}{$\begin{array}{l}\text { Total safety benefit } \\
\quad(\text { million } \$)\end{array}$} \\
\hline & $\begin{array}{c}\mathrm{RA}^{\text {opt_ }} \\
\mathrm{I}\end{array}$ & $\begin{array}{l}\mathrm{RA}^{\text {rank }}- \\
\text { II }\end{array}$ & $\begin{array}{c}\text { RA }^{\text {opt_ }} \\
\text { III }\end{array}$ & $\begin{array}{l}\mathrm{RA}^{\text {opt }} \\
\mathrm{I}\end{array}$ & $\begin{array}{l}\mathrm{RA}^{\text {rank }} \\
\quad \text { II }\end{array}$ & $\begin{array}{c}\mathrm{RA}^{\text {opt. }} \\
\text { III }\end{array}$ & $\begin{array}{c}\mathrm{RA}^{\text {opt. }} \\
\mathrm{I}\end{array}$ & $\begin{array}{l}\mathrm{RA}^{\text {rank }} \\
\text { II }\end{array}$ & $\begin{array}{c}\mathrm{RA}^{\text {opt_ }} \\
\text { III }\end{array}$ \\
\hline Ontario & 91 & 91 & 102 & 5.74 & 5.74 & 6.14 & 114.74 & 114.74 & 122.81 \\
\hline Alberta & 30 & 30 & 26 & 1.47 & 1.42 & 1.33 & 29.47 & 28.36 & 26.63 \\
\hline Quebec & 27 & 27 & 33 & 1.96 & 1.93 & 2.16 & 39.10 & 38.64 & 43.11 \\
\hline Saskatchewan & 26 & 26 & 23 & 1.46 & 1.41 & 1.41 & 29.20 & 28.20 & 28.20 \\
\hline Manitoba & 16 & 18 & 18 & 1.27 & 1.21 & 1.35 & 25.48 & 24.20 & 26.99 \\
\hline British Columbia & 15 & 15 & 11 & 0.90 & 0.87 & 0.77 & 18.05 & 17.40 & 15.35 \\
\hline New Brunswick & 4 & 4 & 0 & 0.10 & 0.10 & 0.00 & 2.07 & 2.00 & 0.00 \\
\hline $\begin{array}{l}\text { Nova Scotia } \\
\text { Newfoundland and }\end{array}$ & 3 & 3 & 0 & 0.06 & 0.06 & 0.00 & 1.25 & 1.20 & 0.00 \\
\hline Labrador & * & * & * & * & * & * & * & * & * \\
\hline Yukon & * & $*$ & * & $*$ & * & * & $*$ & $*$ & * \\
\hline North West territories & * & * & * & $*$ & * & * & $*$ & * & $*$ \\
\hline Total & 203 & & 213 & 12.97 & 12.74 & 13.15 & 259.36 & 254.74 & 263.08 \\
\hline
\end{tabular}

* no priority sites 


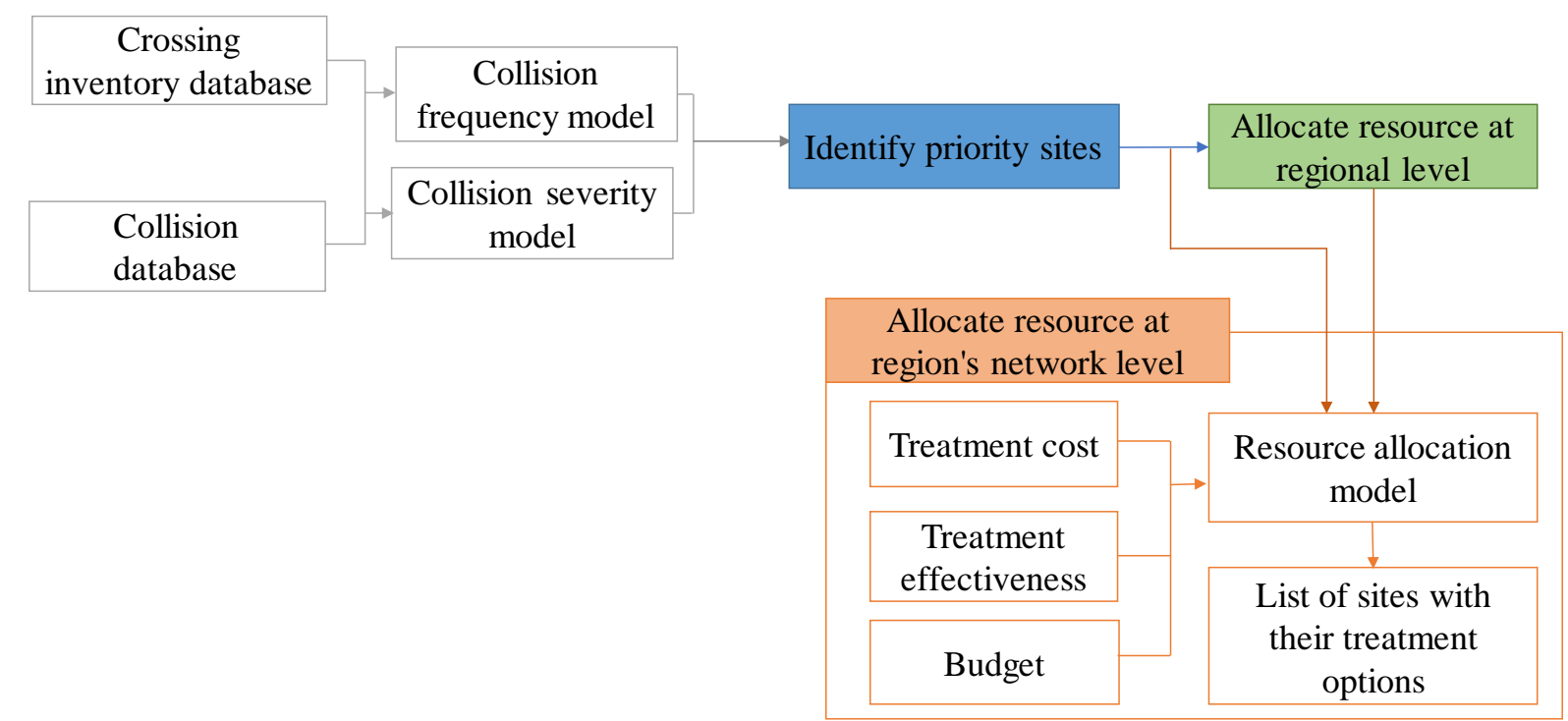

Figure 1: Framework for resource allocation at grade crossings.

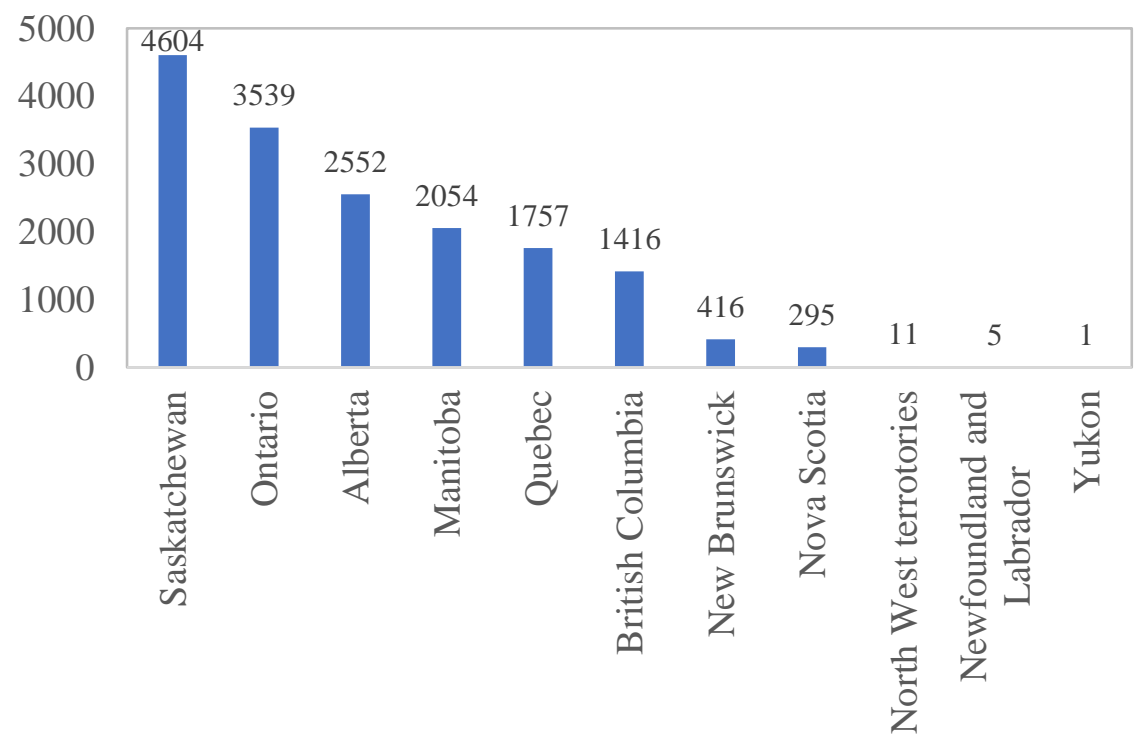

Figure 2: Distribution of crossings across Canadian provinces and territories (of 16,650 crossings being considered) 


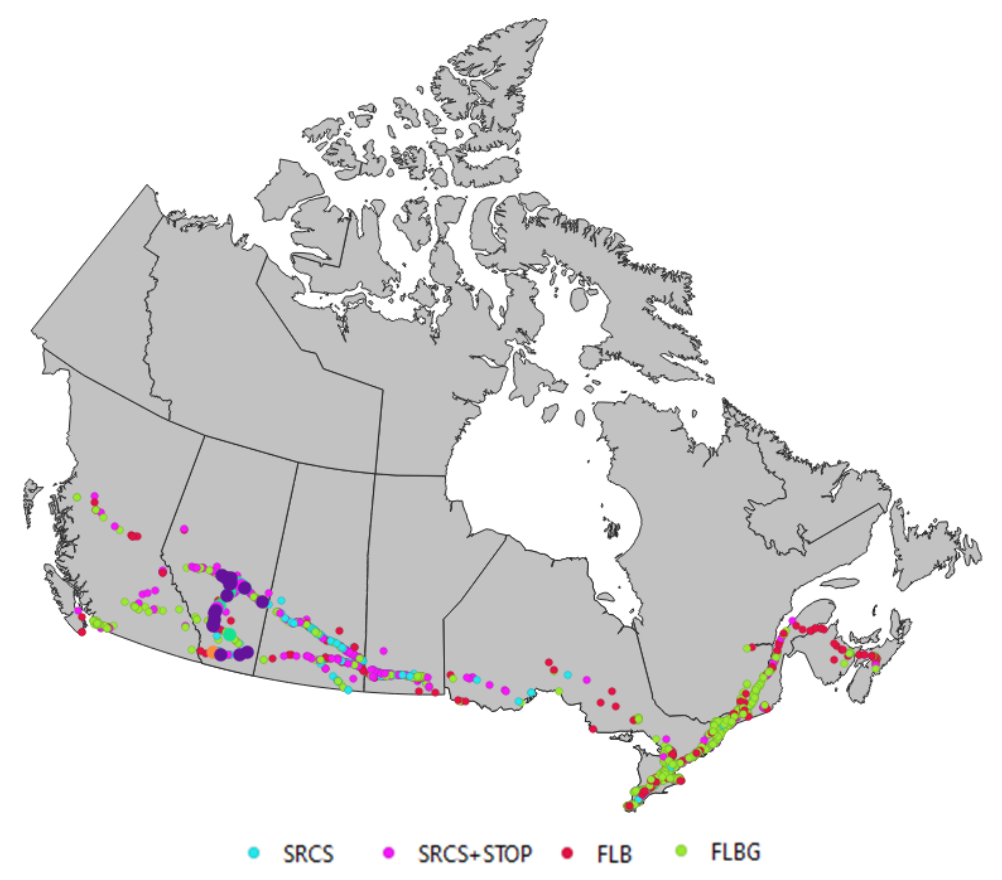

Figure 3: Location of priority sites.
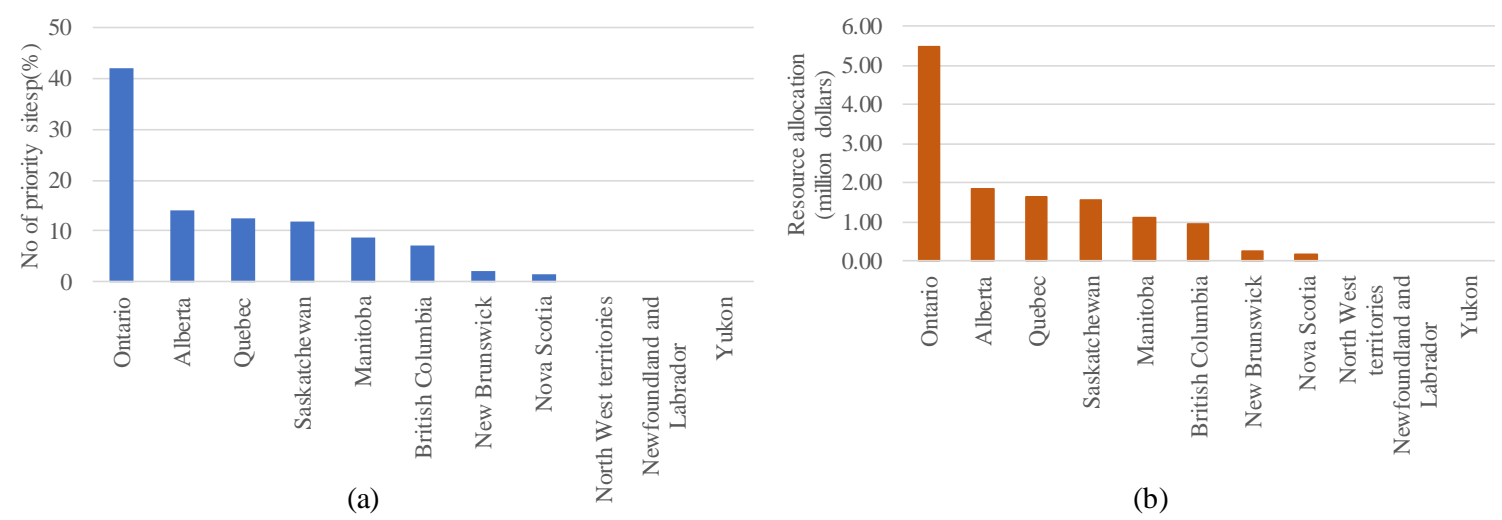

(b)

Figure 4: Distribution of (a) number of priority sites and (b) federal funding across the provinces. 


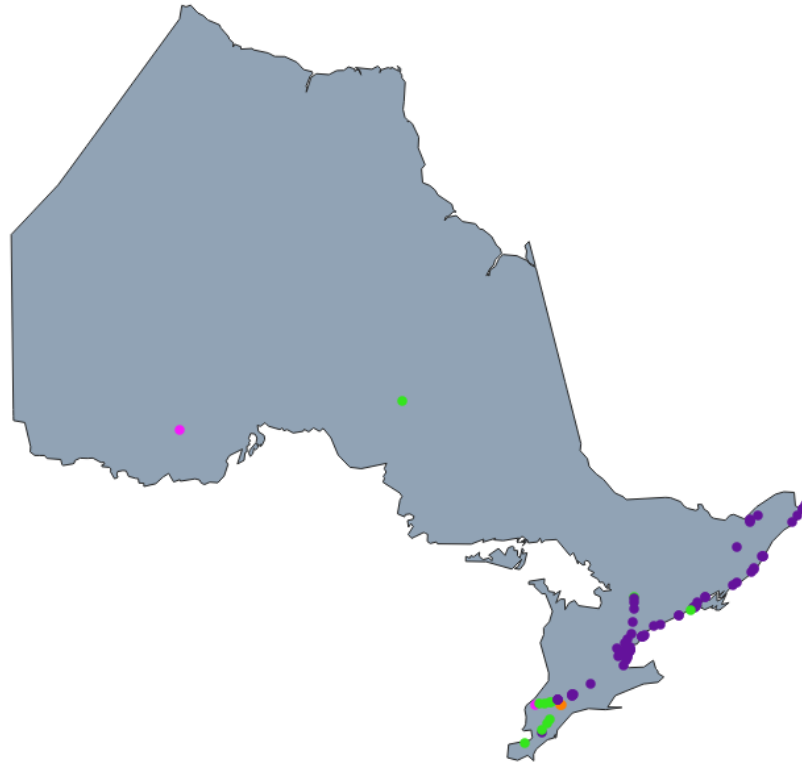

(a)

- Passive to FLB

Passive to FLBG

FLB to FLBG

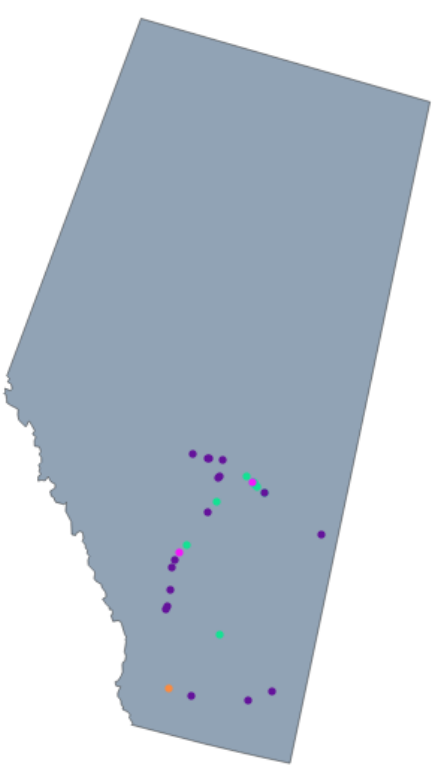

(b)

Figure 5: Location of crossing sites for safety improvement for two provinces: a) Ontario and b) Alberta.

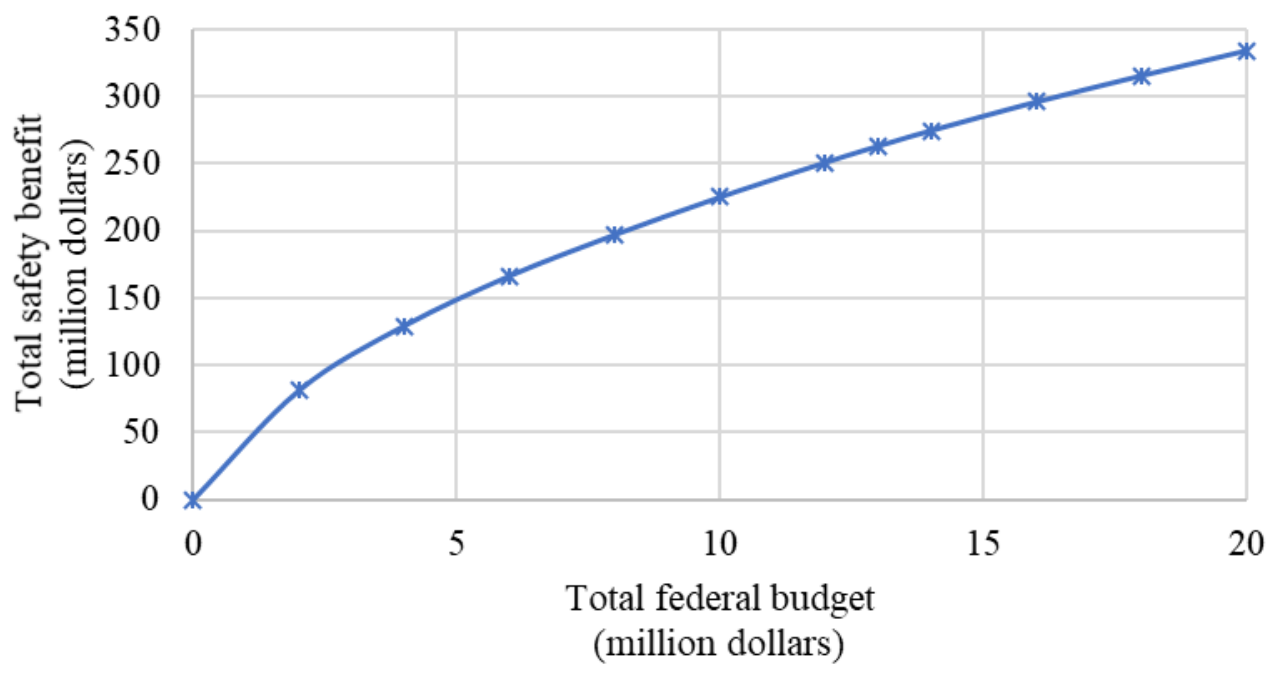

Figure 6: Total expected safety benefit as a function of available budget (investment) 